\title{
Differential recall of problem names and clues as a function of problem solution or nonsolution
}

\author{
ROBERT A. BOTTENBERG, MELVIN H. MARX, and EDWARD J. PAVUR, JR. \\ University of Missouri, Columbia, Missouri 65201
}

\begin{abstract}
An attempt was made to relate the Zeigarnik effect to the broader field of learning. It was assumed that the consequence of task interruption is motivation persistence, assumed to be operative in the classic Zeigarnik situation, and that the consequence of task completion is motivation reduction, generally regarded to be operative in a learning situation. The relative influence of each of these factors on unexpected recall was assessed by comparing two recall measures for a series of problem tasks, some of which had been interrupted and some completed. The first measure was the standard recall of problem names. A clue was introduced in the context of each problem task in order to make possible the second recall measure, which involved responses in the final phase of the total problem task behavior sequence. The recall of clues made possible a more direct test of the influence of reward as a function of task completion. A total of 106 college students were unexpectedly tested for recall of 17 problem names and clues. The results indicated a reliable preferential recall of clues from completed rather than interrupted problems and a slight, but unreliable, preferential recall of problem names from completed rather than interrupted problems.
\end{abstract}

The Zeigarnik phenomenon, first reported by Zeignarik (1927) and confirmed by subsequent investigators (e.g., Marrow, 1938; Pachauri, 1935; Prentice, 1944), refers to the greater relative frequency of recall of interrupted, rather than completed, activities. This result has been interpreted in terms of the persistence of dynamic "tension systems" (Lewin, 1935) corresponding to the interrupted tasks. An unresolved tension system was presumed to carry greater "energy" to the recall situation than one whose energy had been discharged by completion of a task. From this viewpoint, the results of the interruption experiments apparently indicate that recall is aided by the persistence of motivation. On the other hand, learning has typically been assumed to involve motivation reduction via reward.

To assess the relative influence of motivation persistence and motivation reduction in determining differential recall, we compared the recall of tasks influenced by motivation persistence (i.e., tasks interrupted before solution). To insure that task completion would involve reward, tasks were needed in which the subject could not use some response already well established in his behavior repertoire to complete the task. Thus, a task completion situation is characterized by: first, motivation to complete the task; second, trial and error behavior; third, symbolic reward and accompanying motivation reduction when the subject completes the task. We refer to the tasks used in the present study as

The first author is now at the Computational Sciences Division, Department of the Air Force, Air Force Human Resources Laboratory, Lackland Air Force Base, Texas 78236. This research was supported in part by Research Career Award 1-K6-MH-22-023 from the National Institute of Mental Health and by Grant DAHC19-74-G-0008 from the U. S. Army Research Institute for the Behavioral and Social Sciences, to the second author. "problem" tasks in order to distinguish them from the more commonly used "performance" tasks in which the subject uses well-established response patterns to carry out the instructions.

It is generally recognized that the greatest effect of motivation reduction is on the behavior segment which leads directly to reward, and that earlier phases of the sequence are affected more diffusely by the same motivation reduction (Cohen, 1953; Hull, 1932). To obtain a more sensitive comparison between motivation reduction and motivation persistence, problem tasks were designed to permit in themselves a very low probability of solution within a restricted time interval. After an interval of trial and error behavior, the subject was given a clue in the context of the problem task. The function of the clue was to evoke a behavior sequence which could decidedly increase the probability of problem task completion. Differences between the materials and methods used in the present experiment and those employed in previous work lead to the prediction of different results. Preferential recall of clues from completed rather than from interrupted problems, as a function of their close association with reward, was predicted.

\section{METHOD}

\section{Subjects}

The experiment was conducted using an 80-min group testing method is six laboratory sections of one or the other of two introductory college psychology classes. Group size ranged from 11 to 27 , resulting in a total of 133 subjects.

\section{Materials}

Seventeen paper and pencil problem tasks were compiled in mimeographed booklets. The cover page, which instructed the students not to open the booklet until requested to do so, was followed by the tasks, one per page. The last two pages were for 
recall, one for recall of problem task names and the other for recall of clues.

A blank line at the top of each task page was provided for the subject to write the name of that task. Below this line was a one or two sentence statement of the problem, and below the statement were the problem materials. Practice space was provided on each page, and there was a clearly designated place near the bottom of the page for the answer.

Each task was designed to elicit trial and error behavior, and was difficult enough to make a correct solution unlikely without the aid of the clue in a time interval of approximately $31 / 2 \mathrm{~min}$. Each problem had a clear-cut solution, so that, when a correct solution was obtained, there would be no doubt concerning the finished nature of that task.

The problems used can be loosely categorized in this way: five mathematical, five cryptogram deciphering, two spatial relation, two anagram, one word completion, one conundrum, and one synonym problem.

\section{Procedure.}

Subjects came to class expecting a routine laboratory assignment. The regular laboratory instructor in each section served as the experimenter in that section. All subjects in a session worked on the same problem at the same time. They were told not to leaf forward or backward in the booklets. When the time allotted for a task had elapsed, the experimenter said, "Stop. Now go to the next problem which is ..." The subjects then wrote the name of the next task at the top of the page on the line provided. The subjects then read the statement and attempted to solve the problem. Approximately $1 \frac{1 / 2}{2} \mathrm{~min}$ before the time allotted for that task had elapsed, the experimenter said, "Here is an additional instruction," and proceeded to give the clue. Both the problem task name and the problem clue were repeated immediately.

Each recall task was introduced by saying, "The next problem has no name. Turn the page and begin." The instructions at the top of each recall page indicated that the subject was to list problem names or clues in any order that they came to mind. Five and one-half minutes were allowed for each recall task.

Of the total 133 subjects tested, the records of 27 were discarded, including 3 foreign students with language handicaps, 7 students who failed to comply with the instructions, and 17 students who completed less than four problem tasks. The minimum of four completed tasks were set because of the possibility that a greater disparity between the number of completed and uncompleted tasks would bias the results of the experiment.

The 17 th task was excluded from the scoring of all subjects. The work of Marrow $(1938 a, b)$ had indicated that the last

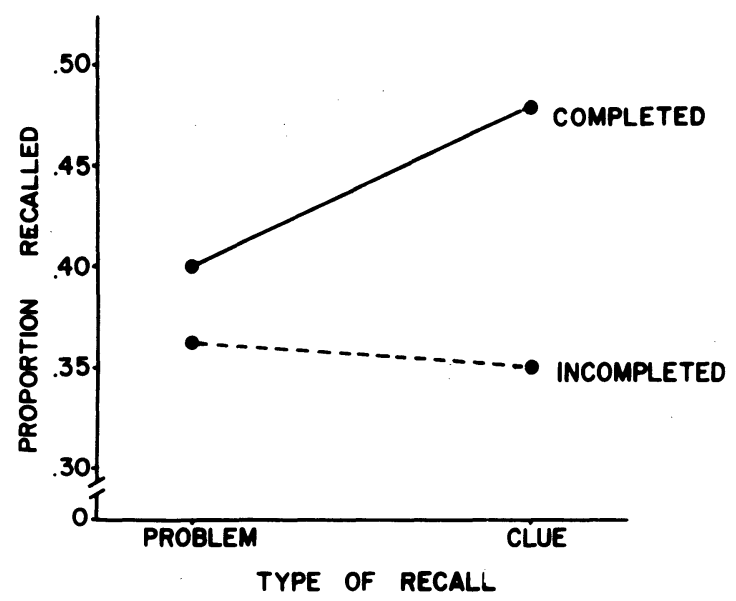

Figure 1. Proportion recalled as a function of completionincompletion and type of recall. task would be recalled by almost all subjects, whether it was completed or interrupted.

All solvable tasks which were not completed were counted as interrupted tasks, since they were functionally interrupted.

\section{Design}

Two forms of each problem were constructed. In the complete (solution-directed) form, the clue enabled the subject to obtain a solution. In the incomplete form, a slight change was made in the statement of the problem or the problem material so that the conditions of the problem made a correct solution unlikely. All of the subjects in a class received booklets in which half of the problems were complete (i.e., a solution was possible with the clue). However, there were two booklet forms or clue sequences, $A$ and $B$, so that the complete problems for half of the subjects were incomplete for the rest and vice versa. The same clue was used for both forms of a given problem. This arrangement not only made it possible to equate the time spent on the complete and incomplete forms of a given problem, but also permitted different subjects in an experimental group to complete a problem and to be interrupted on that problem under constant experimental conditions.

The design also included an instructional variable which shall be referred to as ego involvement. This variable was included for control purposes, due to the possibility that personality variables might operate differently to produce opposite directions of preferential recall under different conditions of stress or ego involvement, as hypothesized by Alper (1946), Glixman (1949), and Rosenzweig (1943). These investigators have argued that interrupted activities are less likely to be recalled if their interruption implies a negative evaluation of the subject's abilities. The operational manipulation was as follows: Subjects in the non-ego-involving condition were presented with the experimental materials in a context designed not to imply a lack of ability by the non completion of a task. These subjects were told that they would be helping the experimenter determine which problems would be appropriate for later experiments. On the other hand, the series of problems given to the subjects in the ego involving condition were referred to as an index of intelligence. Furthermore, the experimenters in this condition read the instructions in a formal manner, asked the subjects to write their names on the test booklets early in the procedure, and made obvious use of a stopwatch during the experiment.

An additional variable, order of recall, was introduced as a control procedure. Subjects in half of the classes were asked to recall clues first and then problem names, while the rest recalled problem names before clues.

Thus, a 2 by 2 by 2 by 2 by 2 mixed factorial design was used. The between-subjects variables were ego involvement, clue sequence, and order of recall. The within-subjects variables were outcome (completion or interruption) and type of recall (problem name or clue).

\section{RESULTS}

The dependent variable was the proportions of problem names or clues recalled. The data were subjected to an arcsine transformation before analysis, the typical procedure for proportional data. Because of unequal numbers of subjects per cell, a regression analysis was employed.

The analyses disclosed reliable main effects for type of recall, $F(1,98)=5.44, p<.02$, and outcome, $F(1,98)$ $=12.22, \mathrm{p}<.001$. More clues than problem names were recalled ( $42.8 \%$ vs. $39.1 \%$ ), and the recall of both problems and clues was greater for solved than for unsolved problems $(45.2 \%$ vs. $36.2 \%)$. 
Figure 1 shows the reliable interaction of Type of Recall by Outcome, $F(1,98)=11.34, p<001$. There was a slight tendency for greater recall of problem names from completed rather than interrupted problems, $F(1,98)=2.98, p<.09$, but a marked tendency for the greater recall of clues from completed rather than interrupted problems, which was highly reliable, $F(1,98)=$ $34.05, \mathrm{p}<.001$.

There was an interaction of Outcome by Clue Sequence, $F(1,98)=12.38, p<.001$. The simple effect of outcome was reliable only for Clue Sequence A, $F(1,98)=22.36, p<.001$. Because of this interaction, the data for the clue sequences were analyzed Since the main effects and interactions involving ego involvement and order of recall were not reliable, these variables were dropped from the analyses. Thus, the resulting analyses of variance were 2 by 2 within-subjects designs. The analysis for Clue Sequence $A$ was based on 61 subjects, and the analysis for Clue Sequence B was based on 45 subjects.

In the Clue Sequence $\mathrm{A}$ analysis, there was a reliable main effect of outcome, $F(1,60)=29.05, p<.001$. The recall of problem names and clues from completed problems was superior to the recall of names and clues from uncompleted problems. The effect of type of recall was not reliable, $F(1,60)=1.14, p<.29$. The interaction of Outcome by Type of Recall was reliable as before, $\mathrm{F}(1,60)=5.03, \mathrm{p}<.03$.

In the Clue Sequence $B$ analysis, the main effect of outcome was not reliable, $F(1,44)<1$. However, there was a reliable effect of type of recall, $F(1,44)=5.03$, $p<.03$, indicating that the recall of clues was superior to the recall of problem names. As in the Sequence A analysis, there was a reliable interaction of outcome by type of recall, $F(1,44)=5.97, p<.02$.

Chi-square analyses of the numbers of subjects preferentially recalling problem names and clues from completed and interrupted problems were also made. With regard to clues, 69 subjects preferentially recalled clues from completed problems, 30 preferentially recalled clues from interrupted problems, and 7 recalled clues from both types of problems equally well. (Subjects showing no preferential recall were excluded in determining expected frequencies.) There was a reliable effect of completion on the number of subjects preferentially recalling clues, $\chi^{2}(1)=15.31, p<.01$. With regard to problem names, 55 subjects recalled more names from completed problems, 45 recalled more names of completed and interrupted problems, and 6 recalled names of completed and interrupted problems equally well. The difference in the number of subjects preferentially recalling problem names was not reliable. $\chi^{2}(1)=1.00, p<.31$.

\section{DISCUSSION}

Task completion and task interruption apparently exerted functionally equivalent influences on the recall of problem names, but task completion had a relatively greater positive influence on the recall of clues. Thus, motivation reduction may be assumed to have exerted a more powerful effect than motivation persistence in the recall of activities which led directly to reward. This conclusion is supported by the fact that each of the subanalyses, done separately for Clue Sequences A and B because of the reliable interaction of Clue Sequence by Outcome, also showed a reliable interaction of Outcome by Type of Recall.

In order to relate the results of the present experiment to previous results, it is necessary to examine the nature of the experimental task material of studies showing the more typical interruption effect. With both her children and adult subjects, Zeigarnik (1927) used such tasks as naming cities, winding string on a reel, reciting a familiar poem, etc. The interruption effect was demonstrated by Pachauri (1935) with such relatively simple tasks as naming a specified number of books, authors, or trees. Marrow $(1938 \mathrm{a}, \mathrm{b})$ performed extensive pre-experimental investigation before he found tasks which college students would preferentially recall when interrupted. The following are examples of those finally selected: connecting scattered numbers with a pencil line, writing a free association word list, cancelling a certain letter each time it appeared on a page.

Such activities as these, when considered in relation to the level of ability of the experimental subjects, in general did not result in random behavior and did not require reorganization of behavior to any marked degree. It seems unlikely that in the performance of these activities there was any selection or emphasis of response patterns. On the other hand, the type of problem material employed in the present study almost invariably elicited random behavior throughout the greater part of the task time interval. It may be hypothesized that completion and interruption have different functions with respect to a "performancetype" task as compared with a "problem-type" task. Two specific hypotheses are suggested:

(1) Completion of a performance task functions primarily to reduce the motivation of the individual toward task continuation. When the organization of the overt and symbolic behavior in the task situation is relatively well established, motivation reduction as a consequence of completion is practically neutral insofar as fixation of this behavior is concerned.

(2) Completion of a problem task reduces the motivation of the individual toward task continuation. But, since there is of necessity a relatively new organization of behavior in the task situation, the motivation reduction as a consequence of completion also serves to fixate this behavior.

In conclusion, the preferential recall of interrupted tasks when the task material involved is performance material can be understood to be a matter primarily of differential degrees of motivation persistence. When material is involved which permits of learning, the present data suggest that the effect of differential motivation in recall is balanced or reversed by the opposite effect of differential reward with respect to completed and interrupted activities. Thus, there will result an equal recall of completed and interrupted activities, or preferential recall of completed activities, especially when these are closely related to the reward function.

\section{REFERENCES}

Alper, T. G. Memory for completed and incompleted tasks as a function of personality: An analysis of group data. Journal of Abnormal and Social Psychology, 1946, 41. 403-420.

Conen, J. The concept of goal gradients: A review of its present status. Journal of General Psychology, 1953, 49. 303-308.

Glixman, A. F. Recall of completed and incompleted activities under varying degrees of stress. Journal of Experimental Psychology, 1949, 39, 281-295.

Hull, C. L. The goal gradient hypothesis and maze learning. Psychology Review, 1932, 34, 25-44. 
LEWIN. K. A dynamic theory of personality. (Translated by D. K. Adams \& K. E. Zener.) New York: McGraw-Hill, 1935.

MARRow. A. J. Goal tensions and recall: I. Journal of General Psychology, 1938, 19, 3-35. (a)

MARROW. A. J. Goal tensions and recall: II. Journal of General Psychology. 1938. 19. 37-64. (b)

Pachauri. A. R. A study of gestalt problems in completed and interrupted tasks. Part II. British Journal of Psychology, 1935. 25. 447-457.
Prentice, W. C. H. The interruption of tasks. Psychological review. 1944, 51. 329-340.

Rosenzweig, S. An experimental study of "repression" with special reference to need-persistive and ego-defensive reactions to frustration. Journal of Experimental Psychology. 1943, 32, 64-74.

ZEIGARNIK. B. Das Behalten erleidigter und unerleidigter Handlungen. Psychologishe Forschungen, 1927, 9, 1-85.

(Received for publication January 26, 1976.) 